\title{
Towards a Growth Mindset Culture in the Classroom: Implementation of a Lesson-Integrated Mindset Training
}

\author{
Helene Zeeb $\mathbb{D}^{1},{ }^{1}$ Julia Ostertag, $^{2}$ and Alexander Renkl ${ }^{1}{ }^{1}$ \\ ${ }^{1}$ Department of Educational and Developmental Psychology, University of Freiburg, 79085 Freiburg, Germany \\ ${ }^{2}$ Max-Born-Gymnasium, 69151 Neckargemünd, Germany \\ Correspondence should be addressed to Helene Zeeb; helene.zeeb@ezw.uni-freiburg.de
}

Received 28 September 2019; Revised 3 February 2020; Accepted 4 February 2020; Published 9 March 2020

Academic Editor: Kirsi Tirri

Copyright $(0) 2020$ Helene Zeeb et al. This is an open access article distributed under the Creative Commons Attribution License, which permits unrestricted use, distribution, and reproduction in any medium, provided the original work is properly cited.

A mindset training aims to strengthen the belief that abilities are malleable (growth mindset), which has proven to be beneficial for learning. Teachers can support the effects of such a training by establishing a classroom culture in line with the growth mindset idea. Yet, previous training programs have mostly been detached from regular lessons. In this study, a physics teacher implemented a mindset training that consisted of explicit training sessions and implicit training phases. In these implicit phases, the teacher enriched ordinary lessons with growth mindset feedback. We investigated the overall effect of this lesson-integrated training on students' beliefs and motivation. Students from two seventh-grade courses participated in the quasi-experimental study $(N=59)$. One course received the mindset training; the other course served as control. We measured growth mindsets about physics abilities, self-beliefs, and motivation before and after the training and six months later. The results indicate that there was a positive and stable training effect on growth mindsets, but no effect on self-beliefs. Regarding motivation, the training buffered the demotivation that occurred without training. We conclude that a mindset training is important when introducing a new and difficult school subject. Furthermore, we consider teachers' involvement as a promising approach to optimize mindset interventions and to encourage a sustained change of instructional practices.

\section{Introduction}

Whether students want to learn depends on whether they believe that they can learn: those who believe that abilities improve with practice (growth mindset) tend to show higher motivation than those who believe that abilities are unchangeable (fixed mindset [1]). Researchers have thus developed training programs to foster growth mindsets among students. However, they yielded mixed results. While some interventions revealed long-lasting effects on students' beliefs, their motivation, and their achievement (e.g., $[2,3]$ ), others had only short-term effects (e.g., [4]). Some interventions failed to affect students' beliefs at all (e.g., [5]). The interventions are usually isolated from regular instruction and delivered by special trainers or online programs. While the effects of educational interventions are often strongest when researchers implement the training (e.g., [6]), the isolation from ordinary lessons may be problematic in the particular case of a mindset training. The reason is that instructional practices-for example, teachers' feedback-influence students' beliefs in a vigorous and permanent manner (e.g., [7]) and may thus influence whether growth mindsets actually take root in the classroom after a training. Indeed, recent studies indicate that the impact of a mindset training interacts with instructional practices and with behavioral norms in the classroom $[3,8,9]$. In the present study, we developed a lesson-integrated training that was implemented by the students' regular physics teacher. In addition to explicit training elements, the intervention included implicit elements within instructional practices. We investigated whether this integrated training fosters the development of growth mindsets, improves self-beliefs, and increases motivation for physics among the students. The study thus sets the base for further studies that compare integrated versus "traditional" nonintegrated interventions.

A growth mindset is defined as the belief that abilities are malleable and can be improved by exercise [1]. This belief has proven beneficial for learning, in contrast to the belief 
that abilities are unchangeable (fixed mindset). For example, a growth mindset supports self-regulated learning and the use of favorable strategies $[10,11]$. Learners with a growth mindset tend to set mastery goals instead of performance goals (e.g., [12]) and show higher engagement for learning (e.g., $[13,14])$. Moreover, mindsets shape learners' subjective well-being, whereby fixed mindsets are associated with negative affect [15]. Overall, a growth mindset seems to help students fulfill their potential and show positive trajectories in their academic performance (e.g., $[2,16]$ ).

Based on these findings, there is broad consensus that growth mindsets should be encouraged in educational settings (e.g., [13, 14, 17-19]). Specific training programs have been developed, which usually consist of just a few sessions, but can yield remarkable effects (e.g., [20]). Many of these mindset interventions are based on an intervention that was originally introduced by Blackwell et al. [2] and further developed by Paunesku et al. [21]. Their program contains three important elements: First, the students receive information about the brain and learning, often via a scientific article or a video. The main message is that the brain can "grow" with practice. Second, the students actively engage with this new information, for example, by discussing examples of how they got smarter by learning or struggling. Third, the students get a task to consolidate a growth mindset. For example, they write an encouraging letter to someone who struggles in school due a fixed mindset. Some researchers have found positive and long-lasting effects of such a mindset training. Most importantly, the training can reinforce positive beliefs in the sense of a growth mindset (e.g., [22, 23]). These changed beliefs can entail further desirable changes, such as affirmative self-beliefs (e.g., $[24,25])$, higher motivation for learning (e.g., [2]), and even better academic performance (e.g., $[3,19,21])$.

However, mindset interventions were not always effective. In a recent meta-analysis, Sisk and colleagues [26] found only a rather small overall effect of mindset interventions. Some studies found positive effects only on beliefs, but not on further outcomes. For example, a training that focused on neuroscientific content supported growth mindsets, but had no impact on motivation or achievement [27]. Similarly, an online training had a stable positive effect on growth mindsets among adolescent girls, but the expected effects on motivation and academic outcomes were absent [28]. Another constraint of previous studies is that some revealed short-term, but no long-term effects. For example, an online intervention strengthened growth mindsets among secondary students immediately after the training, but this effect declined until the follow-up assessment [29]. Likewise, Orosz et al. [4] and Medina-Garrido and León [30] report effects of a mindset training on students' beliefs, which disappeared after a while. Finally, there are interventions that failed to affect students' beliefs at all. A mindset training that was delivered to more than six hundred students did neither affect their beliefs about abilities, nor their effort beliefs or self-efficacy beliefs [5].

There are certainly various reasons why mindset interventions fail. One reason may be that traditional interventions often neglect the students' daily instructional experiences in school. All studies mentioned above have in common that the intervention was somewhat isolated from regular lessons. In most of the studies, the students worked with an online program that delivered the mindset training $[5,28,29]$. Such programs have received a lot of attention, as they are easy to implement, standardized, and scalable. However, the mixed findings indicate that their application in practice does not automatically succeed. In those studies that did not use online programs, the intervention took place as an extra course aside from regular instruction and was provided by specific trainers. For example, in the study by Dommett et al. [27], the intervention was delivered by an online program or specifically trained teachers, and in the study by Orosz et al. [4], the students joined an additional homeroom class and were trained by randomly assigned teachers. The authors argue that an intervention embedded into the school context may have been more efficient. Likewise, Dweck and Yeager [31] report in their review of mindset research that short interventions are not enough; instead, educators have to create growth mindset cultures to induce a persistent change. In the following, we will elaborate on the impact of instructional practices on a mindset training.

Teachers' instruction shapes students' beliefs and, subsequently, students' academic performance (e.g., [7, 32-34]). A well-known mechanism is that teachers communicate their expectations towards students implicitly and thereby influence their development (e.g., $[35,36])$. With regard to students' mindsets, certain instructional practices are known to reinforce fixed mindsets in the class. For example, praise for intelligence supports beliefs in innate talents and can undermine motivation and achievement [37]; comforting feedback such as "not everybody can be good at this" can demotivate students [7]; and subtle cues in teachers' feedback that refer to stable traits can impair students' beliefs and motivation [38]. In contrast, there are instructional practices that reinforce growth mindsets in the classroom. According to Rissanen et al. [39], the core principles of such a "growth mindset pedagogy" are: support individual learning processes (e.g., recognize and overcome individual barriers to learning), promote mastery goals (e.g., avoid comparisons of students' performance), be persistent (e.g., communicate high expectations and accept no helpless behavior), and foster process orientation (e.g., praise favorable strategies and effort). Likewise, Boaler [40] suggests that teachers should set up classroom norms which support growth mindsets. In particular, teachers should communicate clearly that everybody can learn and improve his or her abilities and that mistakes and questions are welcome in the classroom because they are valuable opportunities to learn. In general, instructional practices and behavioral norms that focus on students' traits and performance are likely to support fixed mindsets, whereas practices and norms that focus on learning processes and development are likely to support growth mindsets (see also [41]). It can thus be assumed that instructional practices and classroom norms influence whether growth mindsets are established in the classroom after a training or not. Three recent studies support this assumption. Schmidt et al. [9] implemented an 
online mindset training and investigated whether the teachers' behavior would affect its effectiveness. Indeed, a teacher who reinforced growth mindsets in daily interaction, for example, by focusing on mastery goals or emphasizing appropriate learning strategies, boosted the training effect. In a study by Chao et al. [8], there was an interaction between the intervention and the way how rewards were given in the classroom. Yeager et al. [3] conducted an extensive study with over 6,000 students and found that an online mindset training improved academic achievement among low-achieving students; however, the training effect was stronger when the existing classroom norms supported challenge-seeking behavior (i.e., the norms were consistent with a growth mindset culture).

An option to take the influence of instructional practices and classroom norms on the success of a mindset intervention into account would be to empower teachers to provide the (explicit) training themselves. Furthermore, they could be trained how to (implicitly) encourage growth mindsets when they teach. A longitudinal study with seventh-graders provides first evidence that such an approach can bear fruit [42]. During a whole school year, a language arts teacher implemented a mindset training in her lessons. On the one hand, she used methods of well-studied mindset interventions, such as introducing the concept of a growth mindset and giving information about the brain. On the other hand, she adapted her regular teaching style to the mindset idea, for example, by providing growth mindset feedback, playing mindset games, or modeling and reflecting on reading strategies. The results showed that the students understood the growth mindset concept and incorporated it in their learning routine. However, there was no systematical investigation of the training effects.

In summary, previous research suggests that the everyday instruction that students experience at school plays an important role for the success of a mindset training. Hence, there is good reason to involve teachers in such a training, with the hope that their support induces a sustained improvement of students' beliefs.

1.1. The Present Study. Mindset interventions can have positive effects on students' beliefs, their motivation, and their learning (e.g., [2]). However, they are not always effective (see [26]). One reason why interventions fail may be that they usually leave out the context in which the students learn (see [4]). A sustained change in students' mindsets could be achieved by encouraging teachers to align their regular instruction with the growth mindset idea and establish helpful norms in the classroom. The aim of the present study was to develop a mindset training that was integrated into regular lessons and delivered by the regular teacher. In contrast to previous studies, the mindset training contained not only classical explicit training elements, but also implicit parts in which the teacher adopted the growth mindset idea into regular physics instruction. We investigated whether this lesson-integrated mindset training would have an overall effect on students' beliefs and motivation.
The training was implemented in seventh-graders' physics lessons. We chose physics as a learning domain because fixed mindsets are very common in science education (e.g., $[43,44])$, whereby physics has the reputation of being particularly difficult (e.g., [45]). Hence, a mindset training may be beneficial to foster motivation for this school subject. We chose seventh-graders as participants because physics is introduced as a new subject in seventh grade in German schools. Mindsets matter most when challenges or transitions arise, and a mindset training is particularly beneficial when implemented around such challenges (e.g., $[10,20])$. We thus regarded the introduction of the new and seemingly difficult subject of physics as an adequate time for the intervention. Moreover, as students' initial interest in science predicts their later interest in a scientific career (e.g., [46]), it seems reasonable to strengthen motivation at the beginning of science education.

The mindset training took five weeks and consisted of two implicit parts and two explicit training sessions. The implicit parts contained physics instruction in which the teacher embedded mindset-friendly elements, such as growth mindset feedback and helpful classroom norms (implicit training). The explicit training sessions included elements from well-established mindset interventions (e.g., [2]) and were provided in addition to regular physics lessons (explicit training). We compared the students who received the training to students who did not receive the training. In terms of outcome measures, we were interested in the development of students' beliefs about physics abilities (growth mindset), their self-beliefs, and their motivation for physics.

We addressed the following hypotheses:

First, in accordance with previous research (e.g., $[2,21,22])$, we expected the mindset training to foster the belief that physics ability is malleable (growth mindset). This belief should increase during the training and remain on a high level after the training. For the students without training, we expected no change of growth mindsets (growth mindset hypothesis).

Second, we expected the mindset training to improve students' beliefs about their own physics abilities. In line with Cohen and Sherman [24]; we assumed that the focus on growth mindsets would strengthen feelings of self-affirmation. Hence, we predicted that self-beliefs would improve during the training and remain on a high level after the training. For the students without training, we expected no change of self-beliefs (selfbeliefs hypothesis).

Third, we assumed that the mindset training would increase motivation, as was the case in previous studies (e.g., [2, 19, 25]). According to Pintrich et al. [47], task value is seen as an indicator for learners' motivation. We thus measured the value that students placed on physics and expected this value to increase during the training and remain on a high level after the training. For the students without training, we expected no change of their motivation (motivation hypothesis). 


\section{Methods}

2.1. Participants and Design. Fifty-nine students from two seventh-grade courses participated in the quasiexperimental study (training course: $n=30 ; 63 \%$ female; age $M=12.64$, $\mathrm{SD}=0.49$; control course: $n=29 ; 55 \%$ female; age $M=12.59$, $\mathrm{SD}=0.69)$. All students went to a German secondary school (Gymnasium) and were taught by the same physics teacher. The physics lessons took part twice a week and the topic of all lessons was acoustics. We randomly assigned the courses to one of two conditions: In the training condition, the students received the combined training (implicit and explicit parts). In the control condition, the students did not receive such a training. We measured students' growth mindsets, self-beliefs, and motivation before the training (baseline assessment), directly after the training (post assessment), and six months after the end of the training (follow-up assessment). Personalized codes were used to ensure anonymity.

2.2. Mindset Training. The training consisted of two implicit parts (part 1 and part 2) and two explicit training parts (sessions A and B; see Figure 1). The two implicit parts lasted four lessons each and were based on growth mindset feedback (see, e.g., [48]) and on the establishment of helpful classroom norms (see [40]). The explicit training consisted of two sessions of 45 minutes and drew on the interventions from Blackwell et al. [2], Aronson et al. [22], and on the ideas from Boaler [40]. Teacher and researchers developed the training program together. Such co-construction combines theoretical and practical expertise and thus helps to design interventions that are theoretically sound and, at the same time, work in the specific context of application (see design research; e.g., [49]).

The first part of the implicit training was an introduction to acoustics. The contents of the lessons were identical in both conditions: generation of sound, size of oscillations, and figures of oscillations. Likewise, the methods were the same for all students: experiments, group discussion, partner work, and individual work. However, the teacher varied the kind of feedback she provided to the students. In the control condition, the feedback had no particular focus, whereas in the training condition, feedback was focused towards fostering growth mindsets. Three main principles, which were later introduced as "classroom norms" (during the explicit training; see Table 1), guided the teacher's ad hoc feedback: (1) everyone can learn physics, (2) mistakes are valuable, and (3) questions are really important. These principles have been described, for example, by Boaler [40]; and they are also consistent with the ideas of a growth mindset pedagogy by Rissanen et al. [39]. Table 2 describes example situations in which the teacher provided feedback according to these principles.

Besides these three main principles that guided the teacher's feedback, the "growth mindset feedback tool" [48] was used as a guideline. This tool contains a detailed list of example sentences that apply to different classroom situations and strengthen growth mindsets. Before starting a new lesson in the training condition, the teacher read through the list and thus internalized the kind of feedback she aimed to give. The tool addresses the following situations:

(i) If a student struggles despite effort: encourage the student to recognize the failure as an opportunity to learn, suggest new strategies to solve the problem, praise the student for investing so much effort, etc.

(ii) If a student succeeds with effort: praise the student's effort and persistence, praise the student's behavior (e.g., time management, strategies), point out how much progress the student has made, etc.

(iii) If a student succeeds without much effort: suggest a task that is more challenging, ask the student to help others with the task, look for another skill that the student can work on, etc.

(iv) If a student does not succeed due to a lack of effort: explore what barriers the student is facing and offer help to overcome them, talk about more attractive learning goals, suggest new strategies, etc.

(v) If a student lacks specific skills to improve: suggest new strategies, give further information, encourage the student to try and not to be afraid of mistakes, etc.

(vi) If a student makes progress: praise the student's strategies and hard work, remind the student of the difficulties at the beginning, point out how much progress the student has made, etc.

The first implicit training phase was followed by the first explicit training session (Session A). Contents and methods of this session are depicted in Table 1. The session started with brainstorming about the brain and learning. Afterwards, the students worked on a neuroscientific article that compared the human brain to a muscle that grows as it is trained. The text had the title "The Trainable Brain" and was translated and adapted from the article "You Can Grow Your Intelligence" by Blackwell and colleagues [2]. The article was split into six sections, whereby each section contained a core message about a growth mindset. Everyone had the task to find the most important sentences in the article and talk over their choices with their neighbor. The entire class then discussed the central messages of the article and found personal examples for learning from effort, struggling, or failing.

The teacher introduced three classroom norms: (1) everyone can learn physics, (2) mistakes are valuable, and (3) questions are really important (see [40]). Afterwards, there was a discussion about the case of a fictive student who has fixed beliefs about physics ability. Finally, every student wrote down intentions for future physics lessons (see Table 3).

The second part of the implicit training consisted of four lessons, in which the students worked in small groups on oscillations. Again, the content of this cooperative learning phase was identical in both conditions. The "jigsaw method" was used as a structure. First, the students worked in groups of five or six students on an experiment (e.g., observe a spring pendulum's shadow on the wall and describe its 


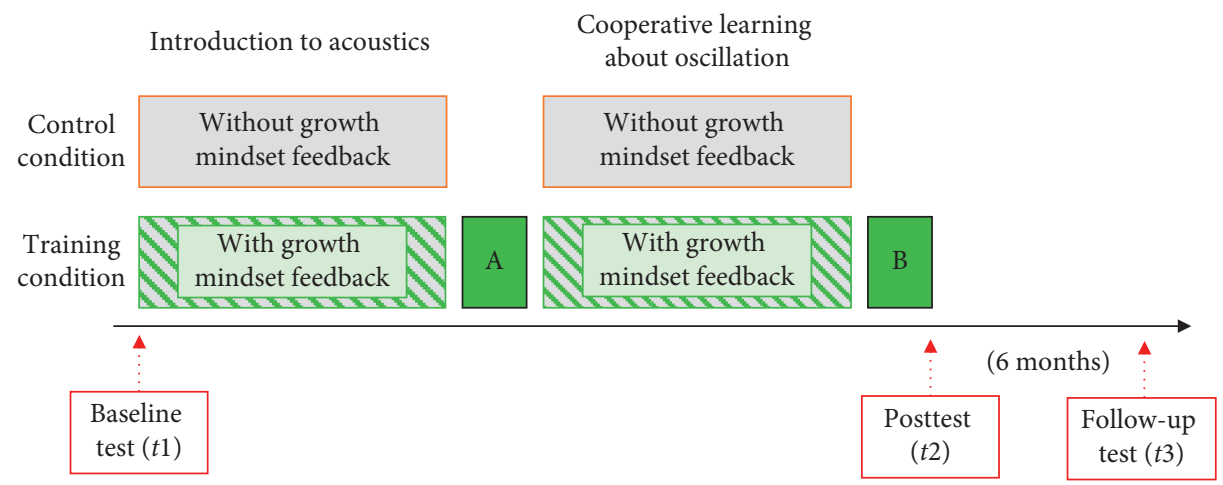

Figure 1: Timescale of the training and the points of assessment. Implicit parts are marked by the cross-hatched areas; explicit training sessions are marked with $\mathrm{A}$ and $\mathrm{B}$.

TABle 1: Training Session A.

\begin{tabular}{ll}
\hline Activity & Description \\
\hline Brainstorming about the brain and learning & (i) The teacher presented a picture of the human brain \\
& (ii) The students brainstormed about the question: "how does my brain learn physics?" \\
(iii) Every student read the neuroscientific article and marked the most important sentence & in each section
\end{tabular}

(iv) The students compared their sentences with their neighbor, justified their choice, and

Teamwork and group discussion about the brain and learning agreed on a solution

(v) The whole class talked about the core messages of the text and discussed how they relate to the students' daily life (moderated by the teacher)

(vi) The teacher wrote the core messages on the board

Introduction of the classroom norms

(vii) The teacher transformed the core messages into three classroom norms: (1) everyone can learn physics, (2) mistakes are valuable, and (3) questions are really important (viii) The teacher explained the norms and pinned them on a poster to the wall

(ix) The teacher introduced the case of the fictive student "Ben," who thinks that there is no point putting effort in learning physics, because nobody in his family was ever good at this

Group discussion about an example case subject

(x) The students made suggestions how to help Ben (e.g., practice a lot, chose more difficult tasks)

Writing good intentions for future physics

(xi) The students wrote down helpful intentions for future physics lessons in their lessons notebooks (e.g., I want to try solving difficult tasks; I want to ask more questions in the lessons; see Table 3).

TABLE 2: Growth mindset feedback.

\begin{tabular}{|c|c|}
\hline Principle & Example situation \\
\hline Everyone can learn physics & $\begin{array}{l}\text { The students were working in pairs on an experiment. The teacher observed that some students } \\
\text { did not put much effort into their work. She motivated them by saying that she believed they had } \\
\text { the potential to learn and achieve much better. }\end{array}$ \\
\hline $\begin{array}{l}\text { Mistakes are valuable; questions are } \\
\text { really important }\end{array}$ & $\begin{array}{l}\text { The teacher asked the students to generate hypotheses about why you can hear a } \\
\text { bumblebee flying, but not a butterfly. A student presented his logical but false } \\
\text { assumption; the different size of the wings might be the reason. Several other } \\
\text { students disagreed and claimed that different movements would cause the different sound. The } \\
\text { first student was confused and asked for a detailed explanation. Guided by the teacher, the } \\
\text { students thus found a conclusive argument for the correct solution. The teacher praised the } \\
\text { student for raising the question and his classmates for their good arguments. She emphasized how } \\
\text { helpful it was to learn from the mistaken assumption. }\end{array}$ \\
\hline
\end{tabular}

oscillation). They read the instructions for the experiment, conducted the experiment, and wrote a protocol together. The teacher provided feedback to the protocols and made copies for each student. Second, the students were assigned to new groups, in which at least one "expert" from each of the five experimental groups was present. Each expert explained his or her experiment to the other students and handed over copies of the experimental protocol. The difference between the two conditions was that in the training condition, there was a specific focus on mindset-friendly 
TABLE 3: Intentions for future physics lessons (samples; translated from German).

\begin{tabular}{ll}
\hline Student & Intention \\
\hline Student A & What I intend to do \\
& $\begin{array}{l}\text { (i) Learn from mistakes } \\
\text { (ii) Carry out experiments } \\
\text { (iii) Train my brain! }\end{array}$ \\
\hline Student B & What do I want to do? I want to solve difficult tasks and not give up \\
Student C & What I intend to do: \\
& (i) ask when there are things I do not understand \\
& (ii) try challenging tasks
\end{tabular}

behavior throughout the group work, whereas in the control condition, there was no specific behavioral focus. The growth mindset focus was realized in the form of a participation quiz [40]. A participation quiz aims at triggering positive interactions between the students and can be applied to any kind of group work. To implement this idea, the teacher first announced that she would pay special attention to the students' behavior throughout the group work. She reminded the students of the classroom norms and described valuable behavior such as respecting and listening to each other, asking questions, engage in meaningful discussions, and learning from mistakes. While the students worked in groups, the teacher observed how they interacted and took notes. At the end, she provided feedback to the entire class and described good and improvable group behavior (see Session B).

The second implicit training phase was followed by the second explicit training session (Session B). Contents and methods of this session are depicted in Table 4. The session started with a reflection on the preceding group work. The teacher first asked the students about their experiences with the classroom norms. For example, one student reported that in her group, they had made a mistake while setting up the experiment, but that this mistake and the subsequent confusion had directed them towards the correct solution. After the students' reflection, the teacher provided detailed feedback about the mindset-related behavior she had observed in the groups (as recommended by Boaler [40]). For example, she reported that she had liked how the students in a group had formulated precise questions and had found good answers together-but that she had not liked how the more dominant students had passed over the quieter students from time to time. To underline her observations, the teacher provided sample situations and student quotes. Afterwards, the students reflected on the intentions they had set in the first training session. Finally, every student wrote an (anonymous) letter to an imaginary student who was struggling with physics. The task was to motivate the student by telling him or her that everybody can be good at physics and to give advice for future learning. Previous studies used this pen pal task successfully (e.g., [22]). Yeager and Walton [20] argue that the success of this task is based on the "saying-is-believing effect": formulating a persuasive message to another person often strengthens one's own persuasion. To encourage thoughtful letters, the teacher announced that she would read each letter and use them for future courses. Two sample letters are depicted in Table 5.

\subsection{Measures}

2.3.1. Growth Mindset. We used three items from the Theories of Intelligence Scale $[1,50]$ to measure students' beliefs about the malleability of abilities, whereby "intelligence" was changed to "physics ability" (e.g., "Everybody has a certain amount of physics ability that can be changed/ cannot be changed"). The students rated the statements on a 5-point scale. We aggregated the items into a mean score; high scores represented a growth mindset. The internal consistency for the scale was low for the baseline test ( $\alpha=0.51)$, but acceptable for the posttest $(\alpha=0.71)$, and the follow-up test $(\alpha=0.83)$. The reason for the low reliability in the baseline test may have been that the students did not have a consistent understanding of "physics ability" yet, as it was a new subject. The results should thus be interpreted with caution.

2.3.2. Self-Beliefs. To assess students' beliefs about their own physics abilities, we used four items from the SelfDescription Questionnaire [51, 52]. The items were adapted to the subject of physics (e.g., "I can solve even difficult tasks in physics"; "I can do well in physics"). The students indicated their agreement on a 6-point scale from 1 (completely disagree) to 6 (completely agree). High scores represented positive self-beliefs. The internal consistency for the scale was good for the baseline test $(\alpha=0.82)$, the posttest $(\alpha=0.82)$, and the follow-up test $(\alpha=0.86)$.

2.3.3. Motivation. Five items assessed the value that the students placed on physics as an indicator for their motivation. The items were taken from the Motivated Strategies for Learning Questionnaire [47]; subscale: task value. We changed "course" to "physics lesson" and "subject matter" to "acoustics" (e.g., "I am interested in the content of acoustics"; "It is important for me to learn the course material in physics"). The students rated their agreement on a 6-point scale from 1 (completely disagree) to 6 (completely agree). High scores represented high value. The internal consistency for the scale was acceptable for the baseline test $(\alpha=0.77)$, the posttest $(\alpha=0.87)$, and the follow-up test $(\alpha=0.87)$. 
TABle 4: Training Session B.

\begin{tabular}{ll}
\hline Activity & Description \\
\hline $\begin{array}{l}\text { Reflection on the group } \\
\text { work }\end{array}$ & $\begin{array}{l}\text { (i) The students reported on situations during the group work in which they had conformed to or deviated } \\
\text { from the classroom norms } \\
\text { (ii) The teacher reported on her observations and provided feedback to each group about their mindset- } \\
\text { related behavior }\end{array}$ \\
\hline $\begin{array}{l}\text { Reflection on the good } \\
\text { intentions }\end{array}$ & $\begin{array}{l}\text { (iii) The teacher reminded the students of the student "Ben" and the suggestions that the students had made } \\
\text { to support him (see Session A) } \\
\text { (iv) The students reflected on their good intentions (see Session A) and provided examples of how they had } \\
\text { conformed to or differed from their intentions }\end{array}$ \\
\hline Pen pal task & (v) Every student wrote a letter to an imaginary student who is struggling with physics (see Table 5) \\
\hline
\end{tabular}

TABle 5: Pen pal task (samples; translated from German).

\begin{tabular}{l}
\hline Student $\quad$ Letter \\
Dear seventh-grader, \\
I am also in seventh grade, but by the time you will have this letter, I'll be already in grade eight. Well, anyway, I should tell you \\
something about physics. So, for me, physics is easy at the moment, and it can be easy for you, too, if you take to heart the \\
following points: \\
Student A Listen well and put your hand up regularly if there is something you don't understand \\
(ii) Look into your physics book more often and repeat everything that you have learned \\
(iii) Don't be afraid to say something/to give a wrong answer. In physics, it is right if you can find a logical justification for your \\
answer \\
(iv) Everybody can learn physics; your brain is trainable! \\
Dear seventh-grader, \\
I know from my own experience that physics doesn't sound very exciting and I have to admit that physics is not my favorite \\
subject, but physics can also be real fun. For example, you carry out cool experiments and most of the time, it is not boring. \\
Maybe you don't believe it but EVERYBODY CAN LEARN PHYSICS AND BE GOOD AT IT! Here are some tips: (1) It helps \\
to pay attention during the lessons (at least pretend to listen-it makes the teacher happy). (2) There is no harm in repeating the \\
content before a test. (3) Always do your homework. (4) Dare to put your hands up, even if you are unsure, and make \\
presumptions. (5) Give a chance to physics! I hope you can learn from that and have a bit of fun! \\
Many greetings, a former student from class 7a
\end{tabular}

2.4. Procedure. The entire procedure of the study is depicted in Figure 1. The study started in the fourth lesson of the school year. First, the teacher explained to the students in both conditions that they would be part of a scientific investigation and administered the pretest at the end of the lesson. In the following four lessons, she introduced the new topic of acoustics via direct instruction, experiments, group work, and individual work. During these lessons, the teacher focused on growth mindset feedback in the training condition, but not in the control condition. Afterwards, the students in the control condition moved to the cooperative learning phase, while the training condition received the first part of the explicit mindset training (Session A). The following four lessons included cooperative learning on oscillation for both conditions. The students worked in small groups according to the jigsaw method. Contents and tasks of the group work were identical in both conditions; however, the teacher focused on growth mindset feedback in the training condition only. In the next lesson, the training condition received the second part of the explicit mindset training (Session B). The teacher then administered the posttest in both courses. The follow-up assessment took place six months after the posttest. During these months, the teacher provided regular instruction with no mindset-related focus to both courses.
2.5. Data Analysis. For the statistical analyses, we used IBM SPSS (Version 22) and R (package lavaan [53]). Structural equation modeling (SEM) was conducted to test the hypotheses. Three models were built to test the hypotheses. Thereby, condition served as a predictor for the three dependent variables (growth mindset, self-beliefs, and motivation) at the three points of assessment. To test specific mean differences over time in SEM, we used the delta method [54]. Note that, in contrast to the usual application of SEM, we created saturated models without latent variables. The models thus produced chi-squared values of zero with zero degrees of freedom; and there were no global fit tests. SEM has the advantage that it allows for estimating missing data. A conventional method such as repeated measures ANOVA would have led to a dropout of 20 students in our data, who had been absent during one of the three assessments. Thus, we used SEM and applied the Full Information Maximum Likelihood (FIML) method to estimate missing values as well as the robust Maximum Likelihood (MLR) estimator. An alpha level of 0.05 was used for all analyses. Preliminary analyses revealed differences between groups prior to the intervention. Such baseline differences often occur in studies with nonrandomized groups and can yield biased results. To address this issue, we conducted additional analyses in which the respective 
baseline variable was added as a further predictor for the post and follow-up variable. The overall baseline mean score is the adjusted mean of both groups prior to the intervention, which needs to be a parameter within the SEM to estimate and test adjusted mean differences within groups over time. Therefore, we defined the overall baseline mean score as an additional model parameter as a weighted sum of the two group means (weighted by relative group sizes).

\section{Results}

3.1. Preliminary Analyses. Table 6 presents the descriptive values for growth mindsets, self-beliefs, and motivation at all three points of assessment.

Preliminary analyses revealed baseline differences between conditions with respect to growth mindsets and selfbeliefs: there were less growth mindsets in the training condition, compared to the control condition, $t(45)=-3.57$, $p=0.001$. Nevertheless, the students in the training condition reported more positive self-beliefs than the control condition, $t(45)=2.43, p=0.019$. Regarding motivation, there were no baseline differences, $t(45)=-0.15, p=0.255$.

Table 7 presents the correlations between all dependent measures. There were no significant correlations between the baseline, post, and follow-up measure of growth mindsets, indicating that these beliefs were not that stable over time. In contrast, the three self-beliefs measures as well as the three motivation measures are significantly associated, indicating that self-beliefs and motivation were more stable over time.

3.2. Growth Mindsets. In the training condition, growth mindsets increased from baseline to post assessment, diff $_{\text {post-baseline }}=0.76, \mathrm{SE}=0.20, p<0.001$, and remained on that level between post assessment and follow-up, diff $_{\text {follow-up-post }}=0.01, \mathrm{SE}=0.25, p=0.979$. The overall development from baseline assessment to follow-up revealed a significant increase, diff follow-up-baseline $=0.76, \mathrm{SE}=0.27$, $p=0.004$. In the control condition, growth mindsets were on a rather high level; however, there were no significant changes between baseline and post assessment or post and follow-up assessment (both $p s>0.701$ ). Likewise, the overall development from baseline assessment to follow-up revealed no substantial change; diff follow-up-baseline $=-0.09, \mathrm{SE}=0.18$, $p=0.618$. After adjusting for baseline differences, we received the same pattern of results: growth mindsets increased in the training condition from baseline to post assessment, diff post-baseline $=0.49, \mathrm{SE}=0.18, p=0.005$, and remained on that level, diff follow-up-post $=-0.06, \mathrm{SE}=0.23$, $p=0.781$. The overall increase was significant, diff $_{\text {follow-up-baseline }}=0.43, \mathrm{SE}=0.21, p=0.036$. In the control condition, there were no substantial changes (all $p s>0.215$ ). These results confirm our hypothesis: among the students who received the training, the belief that physics ability is malleable (growth mindset) increased during the training and remained on a high level. Among the students without training, however, growth mindsets did not change throughout the study. Figure 2 displays the trajectories of growth mindsets in both conditions.
3.3. Self-Beliefs. There was an unexpected decline in students' self-beliefs, whereby the students in both conditions showed a similar pattern. From baseline to post assessment, self-beliefs did not change neither in the training condition, diff $_{\text {post-baseline }}=-0.24, \mathrm{SE}=0.16, p=0.134$, nor in the control condition, $\quad \operatorname{diff}_{\text {post-baseline }}=-0.29, \quad \mathrm{SE}=0.20$, $p=0.151$. From post assessment to follow-up, there was a further descriptive, but non-significant decline in the in the training condition, diff $_{\text {follow-up-post }}=-0.35, \mathrm{SE}=0.19$, $p=0.067$, and in the control condition, diff $_{\text {follow-up-post }}=-0.20, \mathrm{SE}=0.19, p=0.289$. However, the overall decline from baseline assessment to follow-up was significant in the training condition, diff $_{\text {follow-up-baseline }}=-0.58, \mathrm{SE}=0.20, p=0.003$, as well as in the control condition, diff follow-up-baseline $=-0.49, \mathrm{SE}=0.19$, $p=0.011$. After adjusting for baseline differences, we obtained the same pattern of results: self-beliefs decreased nonsignificantly from baseline to post assessment and from post assessment to follow-up (training condition: both $p s>0.078$; control condition: both $p s>0.067)$. The overall decrease was significant in the training condition, diff $_{\text {follow-up-baseline }}=-0.50, \mathrm{SE}=0.21, p=0.016$, and in the control condition, diff follow-up-baseline $=-0.57, \mathrm{SE}=0.19$, $p=0.003$. Figure 3 displays the trajectories of self-beliefs in both conditions: over time, students' conception of their physics abilities decreased substantially in both conditions. Thereby, the students in the training condition reported generally higher self-beliefs as compared to the students in the control condition. These findings do not support our hypothesis that the training would increase students' selfbeliefs.

3.4. Motivation. Motivation did not change in the training condition, neither from baseline to post assessment, $\operatorname{diff}_{\text {post-baseline }}=-0.04, \mathrm{SE}=0.14, p=0.795$, nor from post assessment to follow-up, diff follow-up-post $=-0.16, \mathrm{SE}=0.16$, $p=0.299$. Furthermore, there was no change from baseline assessment to follow-up, $\operatorname{diff}_{\text {follow-up-baseline }}=-0.20$, $\mathrm{SE}=0.19, p=0.303$. In the control condition, motivation decreased from baseline to post assessment, diff $_{\text {post-baseline }}=-0.55, \mathrm{SE}=0.13, p<0.001$, and remained unchanged from post assessment to follow-up, $\operatorname{diff}_{\text {follow-up-post }}=-0.11, \mathrm{SE}=0.15, p=0.464$. The overall decline in motivation from baseline assessment to follow-up was significant, $\operatorname{diff}_{\text {follow-up-baseline }}=-0.44, \mathrm{SE}=0.16$, $p=0.006$. Figure 4 displays the trajectories of students' motivation: while motivation remained on a relatively high level in the training condition, it decreased enormously in the control condition between the baseline test and the posttest. As we expected motivation to increase through the training, these findings contradict our hypothesis. However, the training was successful in that it buffered the students' demotivation during the first physics lessons.

\section{Discussion}

This study sought to develop and implement a lesson-integrated mindset training. The training contained not only 
TABLE 6: Means (and standard deviations) of the dependent variables for both conditions.

\begin{tabular}{|c|c|c|}
\hline Variable & Training condition & Control condition \\
\hline \multicolumn{3}{|c|}{ Growth mindset } \\
\hline Baseline & $3.14(0.82)$ & $3.93(0.70)$ \\
\hline Post & $3.89(0.92)$ & $3.90(0.85)$ \\
\hline Follow-up & $3.93(0.89)$ & $3.84(0.84)$ \\
\hline \multicolumn{3}{|l|}{ Self-beliefs } \\
\hline Baseline & $4.52(0.84)$ & $3.88(0.94)$ \\
\hline Post & $4.29(0.74)$ & $3.56(1.20)$ \\
\hline Follow-up & $3.83(1.14)$ & $3.39(1.11)$ \\
\hline \multicolumn{3}{|l|}{ Motivation } \\
\hline Baseline & $4.10(0.79)$ & $4.36(0.79)$ \\
\hline Post & $4.07(0.83)$ & $3.75(1.04)$ \\
\hline Follow-up & $3.88(0.92)$ & $3.91(1.00)$ \\
\hline
\end{tabular}

TABLE 7: Correlations between the dependent variables.

\begin{tabular}{|c|c|c|c|c|c|c|c|c|c|}
\hline Variable & $1 \mathrm{a}$ & $1 \mathrm{~b}$ & $1 \mathrm{c}$ & $2 \mathrm{a}$ & $2 b$ & $2 \mathrm{c}$ & $3 a$ & $3 b$ & $3 c$ \\
\hline (1a) Baseline growth mindset & - & & & & & & & & \\
\hline (1b) Post growth mindset & 0.26 & - & & & & & & & \\
\hline (1c) Follow-up growth mindset & 0.11 & 0.19 & - & & & & & & \\
\hline (2a) Baseline self-beliefs & -0.09 & 0.18 & 0.12 & - & & & & & \\
\hline (2b) Post self-beliefs & -0.08 & 0.26 & 0.25 & $0.63^{* *}$ & - & & & & \\
\hline (2c) Follow-up self-beliefs & 0.02 & $0.31^{*}$ & $0.37^{*}$ & $0.58^{* *}$ & $0.65^{* *}$ & - & & & \\
\hline (3a) Baseline motivation & 0.28 & 0.22 & $0.47^{*}$ & 0.10 & 0.11 & $0.35^{*}$ & - & & \\
\hline (3b) Post motivation & -0.03 & $0.32^{*}$ & $0.36^{*}$ & $0.30^{*}$ & $0.44^{*}$ & $0.41^{*}$ & $0.68^{* *}$ & - & \\
\hline (3c) Follow-up motivation & -0.07 & 0.18 & $0.53^{* *}$ & 0.25 & $0.48^{*}$ & $0.57^{* *}$ & $0.49^{*}$ & $0.69^{* *}$ & - \\
\hline
\end{tabular}

Notes. ${ }^{*} p<0.05 ;{ }^{* *} p<0.01$.

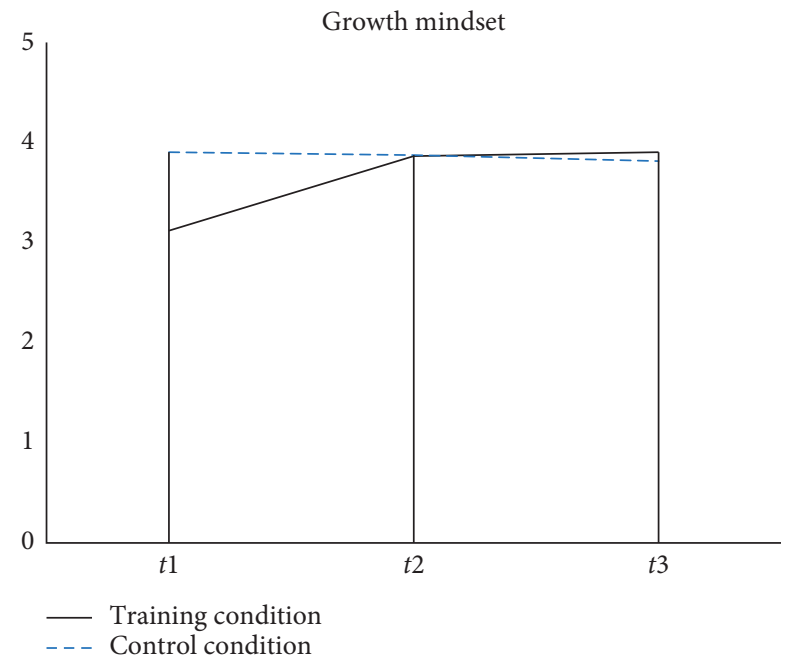

FIgURE 2: Development of growth mindsets in both conditions.

explicit training elements, but also implicit parts in which the teacher aligned regular physics instruction with the growth mindset idea. We explored whether such an integrated training would affect the students' beliefs and motivation. Our results show that the training was partly successful: as expected, growth mindsets were strengthened during the training. This positive effect lasted until the follow-up assessment half a year after the training. However, the training was unsuccessful regarding the students' beliefs about their own abilities. These beliefs decreased over time, independent from whether the students participated in the mindset training or not. Regarding motivation, the results are encouraging insofar as the training buffered the downward trend that occurred in the control group. On the basis of our findings, future studies should unravel the added value of integrating mindset interventions into regular lessons-compared to "traditional" nonintegrated interventions.

4.1. Training Effects on Beliefs and Motivation. The training supported the belief that abilities are malleable. This result is in line with former studies (e.g., $[2,23,28]$ ) and demonstrates once again that mindsets can be trained. In contrast to studies in which effects disappeared after a while (e.g., $[4,29,30]$, we found the positive effect on growth mindsets to last. We consider the theory-based elements from former interventions to be the basis of this sustained effect (e.g., neuroscientific workshop, pen pal task). In addition, we argue that our new approach-aligning regular instruction with the mindset idea-may have deepened the training impact. Our study thus extends the findings from Strahan et al. [42] and provides further positive evidence for a lessonintegrated mindset training.

However, the training had no effect on self-beliefs. In other words, the belief that abilities can (generally) change did not automatically translate into a more positive conception of students' own physics skills. Our training thus failed to induce the positive cycle, in which a growth mindset leads to increased self-affirmation [24]. In contrast, we observed that the perception of one's own skills declined among all students over time. A similar pattern has been reported in previous research. For example, students 


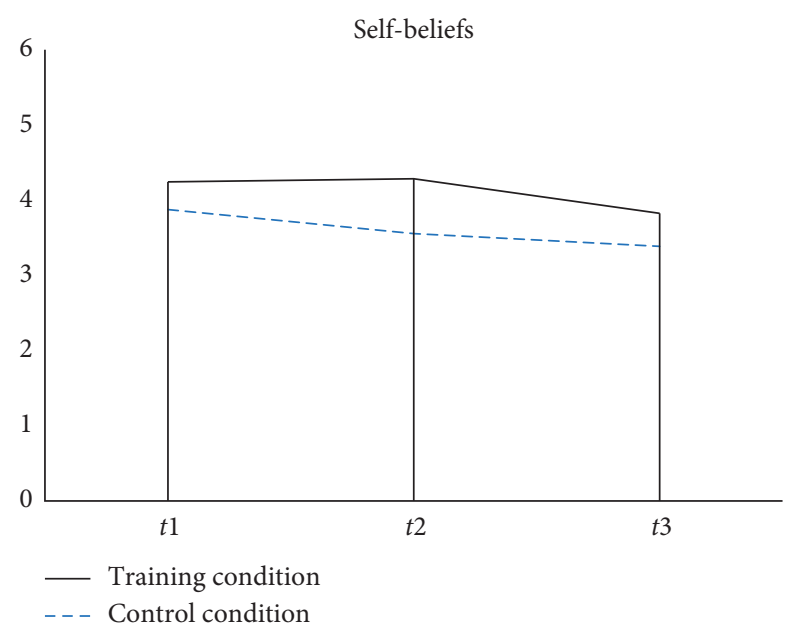

FIGURE 3: Development of self-beliefs in both conditions.

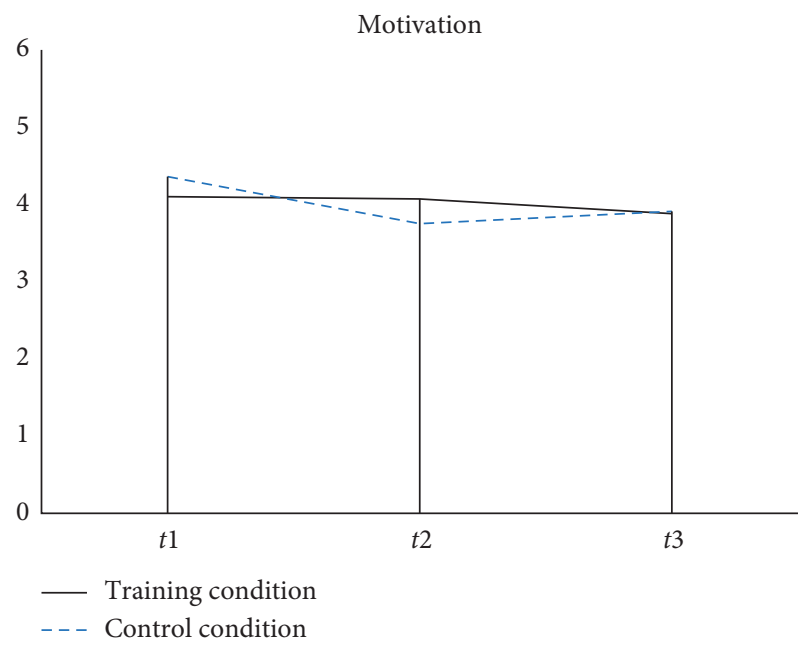

FIgURe 4: Development of motivation in both conditions.

experienced strong declines in perceived skill across the school year, which were only partly buffered by a mindset training [25]. Fredericks and Eccles [55] argue that such downward trend occurs naturally as competitiveness and social comparisons at school become stronger over time. To explain the negative trend in our data, it is thus important to consider how students develop beliefs about themselves (see [56]). We assume that at the beginning, the students had no reference to judge their physics skills, as they had no experience with the demands in physics. The high self-evaluations may resemble an optimistic view on the new subject. As the students became more familiar with physics, they may have adjusted their evaluations. The descriptive values confirm this assumption, as students' agreement rates decreased particularly for those items that referred to physics tasks. For example, the students' confidence in being able to solve difficult tasks decreased. These findings may explain why students' self-evaluations decrease after introducing a new subject in school. Unfortunately, our mindset training was not powerful enough to buffer this downward trend.

With respect to motivation, the training had a "levelingoff" effect. In contrast to the students who received the training, the students without training experienced a substantial decline in their motivation for physics. Such demotivation has often been reported in educational research, especially when students move through adolescence (e.g., [55, 57, 58]). Particularly, in the sciences and in mathematics, there is a typical drop in students' interest and their intrinsic motivation over time (e.g., $[59,60])$. A mindset training can induce an opposite trend and increase motivation (e.g., [19]); however, there are mixed findings. For example, Schmidt et al. [25] found their training to raise interest only among ninth-graders, but not among seventhgraders. Dommett et al. [27] found no training effect on motivational measures at all. Thus, it seems noteworthy that our intervention was successful in supporting the students to maintain their motivation. The positive attitudes towards physics and learning may have reinforced students' interest as well as their value beliefs, which are likely to foster persistence and future learning.

In summary, our results are encouraging as they show that the integrated training that we developed was largely fruitful. Especially with respect to the methodological difficulties of field studies (see Section 4.2), the observed effects on students' mindsets and their motivation can be considered a success. In the next step, it would be interesting to break down the overall training effect by separating the explicit and the implicit training. For this purpose, experimental studies are needed, which compare students who receive only the explicit training to students who receive the combined training. Such studies could show whether growth mindset instruction does indeed boost the effect of a nonintegrated mindset training. Furthermore, the optimal role of the teacher should be clarified. Teachers have a substantial impact on the norms and beliefs in the classroom (e.g., [34, 61]) and are therefore the main actors who can create a growth mindset culture in the long term. Yet, researchers are often better trainers than teachers (e.g., [6]). Particularly, in the case of a mindset intervention, there is the risk that teachers who have not fully understood and internalized the growth mindset idea communicate a "false growth mindset" to their students [31]. For example, if teachers tell their students that everybody can learn physics without providing appropriate strategies, resources, and guidance, the students might become even more frustrated if they fail. In the study by Rissanen et al. [39], a teacher who tended to have a more fixed mindset herself was able to implement growth mindset instruction in her class, but also showed signs of trait-focused thinking and did not actively counter the students' fixed mindsets. Hence, before implementing a mindset training, teachers should confront their own beliefs and ensure that they are able to communicate a growth mindset to their students. Another possibility would be that both researchers and teachers deliver the mindset intervention. Perhaps, a combined training is most effective when a specific trainer implements the explicit training and the regular teacher implements the implicit training parts. We suggest that these open questions should be investigated in follow-up studies. Generally, more experimental studies are needed 
to get insight into what training elements work best and how to combine them sensibly in future interventions.

4.2. Limitations. Our study has several limitations. First, there are restrictions regarding our sample. The sample size was relatively small, which reduces the power of the statistical analyses. Especially, the use of SEM usually requires a bigger sample size. However, the models that we built were saturated and contained only manifest variables. Thus, as no latent variables had to be estimated, the existing data should be sufficient. The reason why we chose SEM was that it allows for estimating missing data and thus prevented a substantial loss of data. Another problem regarding our sample was that there were substantial baseline differences between groups. Particularly, the prior differences regarding growth mindsets seem problematic. As the control condition held high beliefs from the beginning, we could have hardly measured further improvement over time (without training). A randomized allocation could have reduced these baseline differences-but was incompatible with our aim to investigate regular classrooms. Fortunately, the differences took two directions, indicating that no group had systematically better preconditions. Future studies on integrated mindset interventions should draw bigger samples to yield reliable results.

Second, the internal validity may be limited. As we conducted the study in the field, it was impossible to standardize all teaching situations. A particular challenge was that there was only one teacher in both groups, which may have led to unwanted experimenter effects. However, the risk of side effects would have been much higher when varying between teachers. Having one teacher teach both courses had the advantage that we could keep all treatment differences between groups as small as possible-aside from the training. Furthermore, we argue that the applied nature of the study and thus higher external validity outweighs the lower internal validity.

Third, the study design limits the interpretations of our findings. As we investigated just the combined training effect, we cannot judge whether adding the implicit training parts to the explicit intervention actually made a difference. Follow-up studies should compare the success of the training with and without the implicit parts. Furthermore, one could criticize that the control group in our study did not receive a control intervention. Instead, we compared the treatment group with a group that received regular lessons. Such a "business as usual" condition is common in evaluation designs, but provides information only about whether the treatment is better than nothing (e.g., [62]). Future studies should include stronger control groups and compare, for example, different elements of the training.

Fourth, there are restrictions regarding the measurement of dependent variables. We used self-report measures that resembled the students' opinions, but neither their actual behavior nor their implicit thoughts. Future studies should include performance indicators such as students' exams, grades, or classroom observations. Furthermore, the scale that we used for measuring growth mindsets entailed methodological problems, which have also been reported in other studies (e.g., ceiling effects, low reliability; see [23, 63]). In particular, the scale's consistency at the first time of measurement was rather low. We assume that it was difficult for the students to form a consistent judgement about physics abilities because physics was a completely new subject. The fact that the reliability increased over time indicates, however, that the students became familiar with the subject and with the construct of physics ability. These pitfalls could be avoided by using not only explicit, but also implicit measures for assessing beliefs (see also [64]).

\section{Conclusion}

This article describes the development and initial implementation of a mindset training that was integrated into physics instruction. Our approach was based on the findings that instructional practices have a strong impact on students' beliefs and consequently affect the success of a mindset training (e.g., [9]). We see great potential in using this impact for enhancing "traditional" growth mindset interventions. The teacher in our study was, therefore, fully involved and enriched the training with mindset-friendly instructional practices. Our results are mixed: we registered a positive and stable effect on students' beliefs about abilities towards growth mindsets. However, there was no effect on their self-beliefs. Regarding motivation, the training was successful insofar as it buffered the demotivation that occurred without training.

With respect to practical implications, we consider three main points: first, a mindset training seems useful when introducing a new school subject. Particularly, in those domains that have the reputation of being difficult, the risk is high that students will lose motivation. By strengthening growth mindsets, a teacher may create a learning atmosphere that supports students to maintain their motivation. Second, there should be an additional focus on students' selfbeliefs when implementing a mindset training. Mindset interventions typically focus on general beliefs about abilities. Our results indicate that these general beliefs do not automatically trigger positive self-evaluations. We thus suggest complementing a mindset training with ideas from self-concept interventions (e.g., [65]). For example, sensitive feedback after students' success or failure could support helpful attributions. Third, we see a substantial benefit from involving teachers in planning and implementing a mindset training. On the one hand, the teachers' expertise helps to optimize the training for the target group. On the other hand, when teachers are encouraged to align regular instruction to the idea of a growth mindset, there is the chance that this mindset-friendly instruction will persist after the training. In this regard, a lesson-integrated training could help to establish a growth mindset culture in the classroom.

\section{Data Availability}

The SPSS data used to support the findings of this study have been deposited in the OSF (Center for Open Science) repository (https://osf.io/d8baj/). 


\section{Conflicts of Interest}

The authors declare that there are no conflicts of interest regarding the publication of this paper.

\section{Acknowledgments}

The authors are thankful to Norman Rose, who supported the statistical analyses of the data. The article processing charge was funded by the German Research Foundation (DFG) and the University of Freiburg through the funding programme Open Access Publishing.

\section{References}

[1] C. S. Dweck, Self-Theories: Their Role in Motivation, Personality, and Development, Psychology Press, Philadelphia, PA, USA, 2000.

[2] L. S. Blackwell, K. H. Trzesniewski, and C. S. Dweck, "Implicit theories of intelligence predict achievement across an adolescent transition: a longitudinal study and an intervention," Child Development, vol. 78, no. 1, pp. 246-263, 2007.

[3] D. S. Yeager, P. Hanselman, G. M. Walton et al., "A national experiment reveals where a growth mindset improves achievement," Nature, vol. 573, no. 7774, pp. 364-369, 2019.

[4] G. Orosz, S. Péter-Szarka, B. Bőthe, I. Tóth-Király, and R. Berger, "How not to do a mindset intervention: learning from a mindset intervention among students with good grades," Frontiers in Psychology, vol. 8, 2017.

[5] P. B. B. Wilkins, Efficacy of a Growth Mindset Intervention to Increase Student Achievement, ProQuest Information \& Learning, Ann Arbor, MI, USA, 2015.

[6] C. Dignath, G. Buettner, and H.-P. Langfeldt, "How can primary school students learn self-regulated learning strategies most effectively? A meta-analysis on self-regulation training programmes," Educational Research Review, vol. 3, no. 2, pp. 101-129, 2008.

[7] A. Rattan, C. Good, and C. S. Dweck, “'It's ok-not everyone can be good at math": instructors with an entity theory comfort (and demotivate) students," Journal of Experimental Social Psychology, vol. 48, no. 3, pp. 731-737, 2012.

[8] M. M. Chao, S. Visaria, A. Mukhopadhyay, and R. Dehejia, "Do rewards reinforce the growth mindset? Joint effects of the growth mindset and incentive schemes in a field intervention," Journal of Experimental Psychology: General, vol. 146, no. 10, pp. 1402-1419, 2017.

[9] J. A. Schmidt, L. Shumow, and H. Kackar-Cam, "Exploring teacher effects for mindset intervention outcomes in seventhgrade science classes," Middle Grades Research Journal, vol. 10, no. 2, pp. 17-32, 2015.

[10] J. L. Burnette, E. H. O’Boyle, E. M. VanEpps, J. M. Pollack, and E. J. Finkel, "Mind-sets matter: a meta-analytic review of implicit theories and self-regulation," Psychological Bulletin, vol. 139, no. 3, pp. 655-701, 2013.

[11] Y. Karlen and M. Compagnoni, "Implicit theory of writing ability: relationship to metacognitive strategy knowledge and strategy use in academic writing," Psychology Learning \& Teaching, vol. 16, no. 1, pp. 47-63, 2017.

[12] F. C. Dinger and O. Dickhäuser, "Does implicit theory of intelligence cause achievement goals? Evidence from an experimental study," International Journal of Educational Research, vol. 61, pp. 38-47, 2013.
[13] K. C. P. Bostwick, R. J. Collie, A. J. Martin, and T. L. Durksen, "Students' growth mindsets, goals, and academic outcomes in mathematics," Zeitschrift für Psychologie, vol. 225, no. 2, pp. 107-116, 2017.

[14] K. Haimovitz, S. V. Wormington, and J. H. Corpus, "Dangerous mindsets: how beliefs about intelligence predict motivational change," Learning and Individual Differences, vol. 21, no. 6, pp. 747-752, 2011.

[15] R. B. King, "A fixed mindset leads to negative affect," Zeitschrift für Psychologie, vol. 225, no. 2, pp. 137-145, 2017.

[16] J. Zhang, E. Kuusisto, and K. Tirri, "How teachers' and students' mindsets in learning have been studied: research findings on mindset and academic achievement," Psychology, vol. 8, no. 9, pp. 1363-1377, 2017.

[17] J. Boaler, "Ability and mathematics: the mindset revolution that is reshaping education," Forum, vol. 55, no. 1, pp. 143152, 2013.

[18] S. H. Cogdill, "Applying research in motivation and learning to music education: what the experts say," Update: Applications of Research in Music Education, vol. 33, no. 2, pp. 49-57, 2015.

[19] D. S. Yeager, C. Romero, D. Paunesku et al., "Using design thinking to improve psychological interventions: the case of the growth mindset during the transition to high school," Journal of Educational Psychology, vol. 108, no. 3, pp. 374-391, 2016.

[20] D. S. Yeager and G. M. Walton, "Social-psychological interventions in education," Review of Educational Research, vol. 81, no. 2, pp. 267-301, 2011.

[21] D. Paunesku, G. M. Walton, C. Romero, E. N. Smith, D. S. Yeager, and C. S. Dweck, "Mind-set interventions are a scalable treatment for academic underachievement," Psychological Science, vol. 26, no. 6, pp. 784-793, 2015.

[22] J. Aronson, C. B. Fried, and C. Good, "Reducing the effects of stereotype threat on African American college students by shaping theories of intelligence," Journal of Experimental Social Psychology, vol. 38, no. 2, pp. 113-125, 2002.

[23] J. Esparza, L. Shumow, and J. A. Schmidt, "Growth mindset of gifted seventh grade students in science," NCSSSMST Journal, vol. 19, no. 1, pp. 6-13, 2014.

[24] G. L. Cohen and D. K. Sherman, "The psychology of change: self-affirmation and social psychological intervention," $A n$ nual Review of Psychology, vol. 65, no. 1, pp. 333-371, 2014.

[25] J. A. Schmidt, L. Shumow, and H. Z. Kackar-Cam, "Does mindset intervention predict students' daily experience in classrooms? A comparison of seventh and ninth graders' trajectories," Journal of Youth and Adolescence, vol. 46, no. 3, pp. 582-602, 2017.

[26] V. F. Sisk, A. P. Burgoyne, J. Sun, J. L. Butler, and B. N. Macnamara, "To what extent and under which circumstances are growth mind-sets important to academic achievement? Two meta-analyses," Psychological Science, vol. 29, no. 4, pp. 549-571, 2018.

[27] E. J. Dommett, I. M. Devonshire, E. Sewter, and S. A. Greenfield, "The impact of participation in a neuroscience course on motivational measures and academic performance," Trends in Neuroscience and Education, vol. 2, no. 3-4, pp. 122-138, 2013.

[28] J. L. Burnette, M. V. Russell, C. L. Hoyt, K. Orvidas, and L. Widman, "An online growth mindset intervention in a sample of rural adolescent girls," British Journal of Educational Psychology, vol. 88, no. 3, pp. 428-445, 2018.

[29] C. Donohoe, K. Topping, and E. Hannah, "The impact of an online intervention (brainology) on the mindset and resiliency of secondary school pupils: a preliminary mixed 
methods study," Educational Psychology, vol. 32, no. 5, pp. 641-655, 2012.

[30] E. Medina-Garrido and J. León, "Improving the perception of intelligence: a short intervention for secondary school students," Electronic Journal of Research in Educational Psychology, vol. 15, no. 2, pp. 377-397, 2017.

[31] C. S. Dweck and D. S. Yeager, "Mindsets: a view from two eras," Perspectives on Psychological Science, vol. 14, no. 3, pp. 481-496, 2019.

[32] A. Cimpian, Y. Mu, and L. C. Erickson, "Who is good at this game? Linking an activity to a social category undermines children's achievement," Psychological Science, vol. 23, no. 5, pp. 533-541, 2012.

[33] W.-U. Meyer, "Paradoxical effects of praise and criticism on perceived ability," European Review of Social Psychology, vol. 3, no. 1, pp. 259-283, 1992.

[34] D. Park, E. A. Gunderson, E. Tsukayama, S. C. Levine, and S. L. Beilock, "Young children's motivational frameworks and math achievement: relation to teacher-reported instructional practices, but not teacher theory of intelligence," Journal of Educational Psychology, vol. 108, no. 3, pp. 300-313, 2016.

[35] J. Hattie, "The applicability of visible learning to higher education," Scholarship of Teaching and Learning in Psychology, vol. 1, no. 1, pp. 79-91, 2015.

[36] R. Rosenthal and L. Jacobson, "Pygmalion in the classroom," The Urban Review, vol. 3, no. 1, pp. 16-20, 1968.

[37] C. M. Mueller and C. S. Dweck, "Praise for intelligence can undermine children's motivation and performance," Journal of Personality and Social Psychology, vol. 75, no. 1, pp. 33-52, 1998.

[38] A. Cimpian, H.-M. C. Arce, E. M. Markman, and C. S. Dweck, "Subtle linguistic cues affect children's motivation," Psychological Science, vol. 18, no. 4, pp. 314-316, 2007.

[39] I. Rissanen, E. Kuusisto, M. Tuominen, and K. Tirri, "In search of a growth mindset pedagogy: a case study of one teacher's classroom practices in a Finnish elementary school," Teaching and Teacher Education, vol. 77, pp. 204-213, 2019.

[40] J. Boaler, Mathematical Mindsets: Unleashing Students' Potential through Creative Math, Inspiring Messages and Innovative Teaching, Wiley, New York, NY, USA, 2015.

[41] E. De Kraker-Pauw, F. Van Wesel, L. Krabbendam, and N. Van Atteveldt, "Teacher mindsets concerning the malleability of intelligence and the appraisal of achievement in the context of feedback," Frontiers in Psychology, vol. 8, 2017.

[42] D. Strahan, K. Hansen, A. Meyer, R. Buchanan, and J. Doherty, "Integrating mindset interventions with language arts instruction: an exploratory study with seventh grade students," RMLE Online, vol. 40, no. 7, pp. 1-15, 2017.

[43] A.-C. Jonsson, D. Beach, H. Korp, and P. Erlandson, "Teachers' implicit theories of intelligence: influences from different disciplines and scientific theories," European Journal of Teacher Education, vol. 35, no. 4, pp. 387-400, 2012.

[44] M. M. Patterson, N. Kravchenko, L. Chen-Bouck, and J. A. Kelley, "General and domain-specific beliefs about intelligence, ability, and effort among preservice and practicing teachers," Teaching and Teacher Education, vol. 59, pp. 180-190, 2016.

[45] F. Ornek, W. R. Robinson, and M. P. Haugan, "What makes physics difficult?," International Journal of Environmental and Science Education, vol. 3, no. 1, pp. 30-34, 2008.

[46] P. M. Sadler, G. Sonnert, Z. Hazari, and R. Tai, "Stability and volatility of STEM career interest in high school: a gender study," Science Education, vol. 96, no. 3, pp. 411-427, 2012.

[47] P. R. Pintrich, D. A. F. Smith, T. Garcia, and W. J. McKeachie, A Manual for the Use of the Motivated Strategies for Learning
Questionnaire (MSLQ), University of Michigan, Ann Arbor, MI, USA, 1991.

[48] Mindset Works, "Growth mindset feedback tool," 2016, https://www.mindsetworks.com/free-resources/.

[49] M. W. Easterday, D. G. Rees Lewis, and E. M. Gerber, "The logic of design research," Learning: Research and Practice, vol. 4, no. 2, pp. 131-160, 2017.

[50] B. Spinath and Schöne, "Subjektive Überzeugungen $\mathrm{zu}$ Bedingungen von Erfolg in Lern- und Leistungskontexten und deren Erfassung," in Diagnostik von Motivation und Selbstkonzept, J. Stiensmeier-Pelster and F. Rheinberg, Eds., pp. 15-27, Hogrefe, Göttingen, Germany, 2003.

[51] H. W. Marsh, "A multidimensional, hierarchical model of self-concept: theoretical and empirical justification," Educational Psychology Review, vol. 2, no. 2, pp. 77-172, 1990.

[52] A. Friedrich, B. Flunger, B. Nagengast, K. Jonkmann, and U. Trautwein, "Pygmalion effects in the classroom: teacher expectancy effects on students' math achievement," Contemporary Educational Psychology, vol. 41, pp. 1-12, 2015.

[53] Y. Rosseel, "Lavaan: an R Package for structural equation modeling," Journal of Statistical Software, vol. 48, no. 2, pp. 1-36, 2012.

[54] G. W. Oehlert, "A note on the delta method," The American Statistician, vol. 46, no. 1, pp. 27-29, 1992.

[55] J. A. Fredricks and J. S. Eccles, “Children's competence and value beliefs from childhood through adolescence: growth trajectories in two male-sex-typed domains," Developmental Psychology, vol. 38, no. 4, pp. 519-533, 2002.

[56] H. W. Marsh, "Verbal and math self-concepts: an internal/ external frame of reference model," American Educational Research Journal, vol. 23, no. 1, pp. 129-149, 1986.

[57] P. Barmby, P. M. Kind, and K. Jones, "Examining changing attitudes in secondary school science," International Journal of Science Education, vol. 30, no. 8, pp. 1075-1093, 2008.

[58] J. A. Fredricks, P. C. Blumenfeld, and A. H. Paris, "School engagement: potential of the concept, state of the evidence," Review of Educational Research, vol. 74, no. 1, pp. 59-109, 2004.

[59] A. E. Gottfried, J. S. Fleming, and A. W. Gottfried, "Continuity of academic intrinsic motivation from childhood through late adolescence: a longitudinal study," Journal of Educational Psychology, vol. 93, no. 1, pp. 3-13, 2001.

[60] A. Krapp and M. Prenzel, "Research on interest in science: theories, methods, and findings," International Journal of Science Education, vol. 33, no. 1, pp. 27-50, 2011.

[61] E. A. Canning, K. Muenks, D. J. Green, and M. C. Murphy, "STEM faculty who believe ability is fixed have larger racial achievement gaps and inspire less student motivation in their classes," Science Advances, vol. 5, no. 2, 2019.

[62] L. R. Peck, Experimental Evaluation Design for Program Improvement, SAGE Publications, Thousand Oaks, CA, USA, 2019.

[63] H. Rieche, T. Leuders, and A. Renkl, "If a student thinks, "I'm not a math person," do preservice teachers notice?," European Journal of Science and Mathematics Education, vol. 7, no. 1, pp. 32-49, 2019.

[64] M. Lüftenegger and J. A. Chen, "Conceptual issues and assessment of implicit theories," Zeitschrift für Psychologie, vol. 225, no. 2, pp. 99-106, 2017.

[65] A. J. O’Mara, H. W. Marsh, R. G. Craven, and R. L. Debus, "Do self-concept interventions make a difference? A synergistic blend of construct validation and meta-analysis," $E d-$ ucational Psychologist, vol. 41, no. 3, pp. 181-206, 2006. 